\title{
Pablo Garrido y su defensa de los derechos laborales de los músicos en Chile (1932-1940)
}

\section{Pablo Garrido's advocacy of musicians' labor rights in Chile (1932-1940)}

\author{
por \\ Eileen Karmy \\ Centro de Estudios Avanzados y Departamento de Artes Integradas, \\ Universidad de Playa Ancha, Chile \\ eileen.karmy@upla.cl
}

En este artículo analizo la figura de Pablo Garrido desde el prisma del trabajo musical, destacando su contribución a la organización gremial de músicos en Chile en la tercera década del siglo XX. Argumento que solo desde una perspectiva que enfatice lo laboral es posible abordar de manera integral la figura y aporte de un músico ecléctico como Garrido, cuyo rol ha sido estudiado fundamentalmente desde la contribución que ha hecho a distintos géneros musicales, sin considerarlo como un trabajador de la música. A partir del análisis a los escritos de prensa que publicó Garrido identifico sus principales preocupaciones y propuestas respecto del trabajo musical, las que desembocaron en el Primer Congreso de Músicos de 1940. Concluyo destacando la complejidad de definir el trabajo musical haciendo alusión a la necesidad de comprender su faz en tanto trabajadores y artistas, en constante tensión.

Palabras clave: Pablo Garrido, trabajadores de la música, sindicatos de músicos, gremio musical, Congreso, arte y trabajo, música en Chile, música del siglo XX.

Through the prism of musical work, this article analyses Pablo Garrido's contribution to musicians' unions in Chile during the nineteen-thirties. I argue that the perspective of musical labour allows understanding an eclectic musician such as Garrido in a comprehensive way, rather than isolated. Prior scholarship has studied his contribution to different genres rather than considering him as a musical worker. By analysing Garrido's newspaper articles, I identify the main problems Chilean working musicians suffered and his proposals to overcome them. All these resulted in the First Musicians' Congress in 1940. I conclude by highlighting the difficulty of defining the musical work and emphasising the need to understand musicians' two-sided identity as labourers and artists in a perennial tension.

Keywords: Pablo Garrido, music workers, musicians' unions, music guild, Congress, art and labour, music in Chile, 20th century music. 


\section{INTRODUCCIÓN ${ }^{1}$}

En este artículo abordo la figura del músico e investigador Pablo Garrido desde una perspectiva que hasta ahora no ha sido estudiada: su rol como activista y defensor de los derechos laborales de los músicos en Chile. Garrido fue un músico multifacético que aportó al desarrollo de distintos géneros en el país, al tiempo que, como investigador, estudió y difundió músicas diversas. Por ello, ha sido relevado fundamentalmente por su aporte al desarrollo del jazz en Chile (Menanteau 2006: 29-39; Cabello 2020: 92-134), por sus trabajos etnomusicológicos y su contribución al folclor y la cueca (Donoso y Tapia 2017: 137; Ramos 2012: 100-101), mientras que su experiencia como compositor de vanguardia ha sido estudiada solo recientemente (Fugellie 2019). Como demostraré a lo largo de este artículo, aunque hasta ahora no haya merecido suficiente atención su aporte como activista de los derechos de los músicos, fue de fundamental importancia. Garrido lideró un movimiento de organización gremial de músicos profesionales durante toda la década del treinta, mientras se configuraba un marco legal de seguridad social y laboral en el país. Este movimiento permitió que los músicos analizaran su particular situación como trabajadores, hicieran propuestas y se encontraran en un congreso nacional en 1940, a partir de ahí se formó la Federación de Músicos de Chile.

Comenzaré el artículo con una sección teórica que inserta la discusión en un debate más amplio y de larga data. Luego, a partir de la trayectoria artística de Garrido, que lo sitúa en el contexto del que fue parte y permite comprender mejor su decisión de dedicarse a la música y sus implicancias. Luego, a partir de sus escritos periodísticos, analizaré el rol que cumplió en la preparación del Primer Congreso de Músicos, que se realizó en Santiago de Chile en 1940, reuniendo a todos los sindicatos de músicos y comités gremiales que en ese entonces había en el país. Detallaré los puntos centrales que discutió Garrido en sus escritos y que sirvieron de base para el Congreso: la necesidad de crear instituciones estatales de patrocinio musical, el impacto de la legislación vigente en el trabajo musical y la necesidad de formación y fortalecimiento de sindicatos de músicos. Finalmente daré cuenta acerca de la realización del congreso, sus participantes, actividades y resultados directos e indirectos, destacando la contribución de Garrido.

Las fuentes primarias utilizadas para esta investigación corresponden a documentos de archivo tanto públicos como privados. Los escritos de Garrido así como su correspondencia y fotografías incluidas en las siguientes páginas están alojados en el Fondo Pablo Garrido (FPG) del Centro de Documentación e Investigación Musical (CEDIM) de la Facultad de Artes de la Universidad de Chile. Los primeros están almacenados en cuatro libros de recortes que consignan la fecha y el nombre del medio de comunicación donde fueron publicados. En el CEDIM también están los documentos correspondientes al Sindicato Profesional Orquestal referidos en este artículo, almacenados en el Fondo Domingo Santa Cruz (FDS). Los documentos de archivo de los gremios de músicos de Valparaíso mencionados aquí incluyen actas de reuniones y asambleas, libros de inscripción y pagos,

1 Este artículo es una ampliación de la ponencia "Sindicalismo musical como defensa de los derechos laborales de los músicos, 1932-1940" presentada por la autora en el Seminario Pablo Garrido y la música del siglo XX, reflexiones en torno a su archivo, realizado en Santiago de Chile el 23 de agosto de 2019, organizado por Karen Donoso e Ignacio Ramos, a quienes agradezco la invitación. La investigación es parte de mi tesis doctoral, realizada con financiamiento de Becas Chile en la Universidad de Glasgow y agradezco a mis profesores Eva Moreda, John Williamson y Martin Cloonan por sus enriquecedores comentarios. Agradezco también a Rodrigo Torres por facilitarme el acceso al Centro de Documentación e Investigación Musical (CEDIM) de la Universidad de Chile y a Álvaro Menanteau por advertirme de su importancia. 
cartas, estatutos, memorias anuales, fotografías y folletos y pertenecen al actual sindicato de músicos de Valparaíso (SIMUPROVAL) ${ }^{2}$. Al momento de acceder a estos documentos ninguno de estos archivos estaba catalogado.

\section{EL ARTE COMO TRABAJO Y LOS DESAFÍOS DEL ASOCIATIVISMO}

La relevancia de este artículo reside en que, solo a partir de una perspectiva integral respecto del rol de este músico, es posible advertir su faceta de trabajador y no solamente de artista. Analizar la música desde el prisma del trabajo permite, por una parte, ahondar en las condiciones laborales de los músicos, independiente de si se dedican a la composición, la interpretación, la investigación o la enseñanza de cualquier género musical. Por otra, permite ampliar y democratizar el alcance que puedan tener los músicos representados en la investigación musical (Miller 2008: 438). Esta perspectiva contribuye a escribir una historia "manteniéndonos alerta de los efectos de la valoración occidental de las obras musicales por sobre el trabajo musical, de la composición por sobre la interpretación"3 (Miller 2008: 439). Esto permite no solo considerar como actores válidos de investigación a los artistas destacados de la historia de la música, sino que también a aquellos que la historiografía ha pasado por alto, o en palabras de la etnomusicóloga Ruth Finnegan, han estado "ocultos a plena vista" (2007: xvii).

En general, la historia de la música "ha presentado a los músicos como artistas, creadores y celebridades y con menor frecuencia como personas haciendo un trabajo" (Cloonan 2014: 12). Concebir a los músicos como personas haciendo un trabajo contribuye a develar facetas que no conocíamos, como en el caso de Pablo Garrido, en que ni su rol como trabajador ni su aporte a la organización gremial de la música han sido tomados en cuenta seriamente, hasta ahora. Al develar esta faceta, su imagen de artista que propone la perspectiva hegemónica (Vera 2015: 108) cambia a una imagen de un trabajador de la música que vivió ciertas condiciones de vida y trabajo y que participó de un movimiento social para mejorar dichas condiciones. Más aún, la perspectiva laboral permite evidenciar cómo las organizaciones musicales han moldeado nuestro propio entendimiento del trabajo musical (Frith 2017: 112) y el de las instituciones públicas que lo regulan (Williamson y Cloonan 2016: 8).

Este artículo que emerge de un estudio de caso de un músico en específico, Pablo Garrido, y su rol en el movimiento gremial de músicos en Chile, se inserta en un debate más amplio y de larga data acerca del arte como trabajo. Hay un creciente corpus de investigaciones acerca del trabajo musical, realizadas en mayor medida en el norte global, que ofrecen marcos teóricos y metodológicos en los que se sostiene este estudio. Ya Howard Becker (1974) planteaba la relevancia de comprender el trabajo artístico como una actividad colectiva, tomando en consideración a todos los actores que participan en la producción de una obra y desentrañando la división social del trabajo artístico (Becker 1982), mientras que Jaques Attali (1985) evidenciaba cómo la aparición del capitalismo industrial cambió la vida laboral de los músicos. Inserta en este debate del arte como trabajo, Angéle DavidGuillou (2009) sugiere que, en el caso de la música, la oposición entre arte y trabajo es única, incluso en el campo artístico, ya que, "a diferencia de la pintura, por ejemplo, esta es originalmente inmaterial y, por tanto, históricamente no ha tenido un valor” (David-Guillou

2 Muchos de estos documentos han sido digitalizados y puestos a disposición en el sitio http:// memoriamusicalvalpo.cl/, por la autora en conjunto con Cristian Molina desde 2015.

3 Esta y las demás traducciones de publicaciones en idiomas distintos al castellano son de la autora. 
2009: 209). Karl Hagstrom Miller (2008) explora las implicancias de que la música parezca como algo que no requiere esfuerzo, sino que talento, goce y genialidad individual más que ser el resultado de un trabajo. Como se ha planteado ampliamente, el problema de esta noción es que puede sostener situaciones de autoexplotación (Gross y Musgrave 2020: 46-47) y conducir a trabajo no remunerado a cambio de exposición o aprendizaje (Brook, O'Brien y Taylor 2020: 136-134).

Además, el trabajo de los músicos ha sido en general de naturaleza independiente e informal, funcionando bajo las lógicas de la gig economy. Este concepto, que podemos traducir como la economía de la tocata o del concierto, se acuñó para referirse a trabajos independientes o de corto plazo, donde los trabajadores se sustentan con diversos empleos de tiempo parcial y no reciben beneficios sociales como sistema de salud (Hook 2015). Lo interesante de esta situación laboral considerada precaria es que, aunque no es exclusiva del campo musical, su nombre proviene de él. Si bien la incorporación de este concepto en el mundo de la economía es relativamente nueva, las nociones de precariedad en labores artísticas son antiguas. Los músicos han trabajado en la gig economy "durante la mayor parte del siglo [veinte] y la naturaleza de su trabajo no ha sido tomada en consideración" (Cloonan y Williamson 2017: 494). Para revertir esta situación, los músicos se han agrupado en asociaciones gremiales, muchas de ellas con el fin de regular sus trabajos o mejorar sus condiciones.

Un antecedente importante a este estudio es la tesis doctoral de Adam Loft (1950) en la que analiza las experiencias de organización de los músicos en Europa y Estados Unidos, incluyendo gremios, sociedades y sindicatos. Angéle David-Guillou (2009) sostiene que la historia de los gremios de músicos debe ser puesta en perspectiva con la historia de las organizaciones obreras, ya que estas "siguen un patrón similar", yendo desde "sociedades mutualistas a sindicatos profesionales más cerrados para avanzar hacia grupos industriales y finalmente, confederaciones" (David-Guillou 2009: 290). Y, en este proceso, la problemática del arte como trabajo releva su importancia. Por ejemplo, como evidencia James Kraft en su estudio acerca de la formación de sindicatos de músicos en Estados Unidos, la mayor dificultad en este proceso fue la propia reticencia de los músicos de concebirse como trabajadores (Kraft 1995: 513). Este punto es tomado en cuenta también por David-Guillou (2007), quien demuestra cómo ciertos gremios de músicos optaron por mantenerse alejados del sindicalismo "por temor a que pudiera empañar la respetabilidad de sus miembros" (David-Guillou 2007: 295).

Manuel Deniz Silva (2017) analiza el modo en que los músicos sindicalizados en Portugal de principios del siglo XX abordaron las contradicciones de su propia posición social en un periodo marcado por el desarrollo de las industrias del entretenimiento. Da cuenta cómo estos músicos se sentían parte del movimiento obrero al tiempo que mantuvieron el ideal de la orquesta sinfónica y de la noción de un arte musical puro y fuera del mercado (Silva 2017). Develando una contradicción similar, en estudios anteriores acerca de la Sociedad Musical de Socorros Mutuos de Valparaíso he mostrado cómo los músicos que fueron parte de ella se identificaron con el movimiento obrero a conveniencia, alejándose de su identidad obrera cuando requerían reforzar su identidad artística (Karmy 2017; Karmy y Molina 2018).

Como veremos en la siguiente sección, Pablo Garrido fue miembro de la Sociedad Musical de Socorros Mutuos y, a partir de ahí, comenzó a desarrollar su faceta de activista y defensor de los derechos laborales de los músicos en Chile. Considerando que Garrido fue un músico ecléctico, que se desempeñó en diversos géneros y ocupó distintos lugares dentro de la división social del trabajo musical, su trayectoria es un caso de estudio interesante, que ofrece abundante información tanto de lo laboral como de las iniciativas asociativas. Al haberse desarrollado como compositor, intérprete, investigador y otras labores al interior del campo musical, su caso refleja la situación de una multiplicidad de músicos trabajando 
en Chile en la misma época, así como en otros contextos de condiciones similares. Conocer cuáles eran las necesidades que Garrido estableció como prioritarias a resolver permite obtener información de las condiciones en que trabajaban los músicos en Chile entre los años veinte y cuarenta. Esto dará luces de un aspecto poco explorado de la historia de la música en Chile, con sus conflictos y tensiones, develando las dinámicas de las organizaciones gremiales de músicos y el perfil de la profesión musical que estas moldearon.

\section{PABLO GARRIDO Y SU DESPERTAR COMO TRABAJADOR DE LA MÚSICA}

Pablo Garrido nació en Valparaíso en 1905 en una familia de artistas e intelectuales, hijo de un profesor y artista visual, y de una profesora de idiomas y de música. Desde temprana edad se desarrolló musicalmente, con clases de piano y canto, participando también en el coro y banda del colegio donde estudiaba, el Mackay School de Viña del Mar. Un grave accidente de tranvía, en enero de 1913, cambiaría su rumbo. Al perder su pierna derecha, debió dejar sus estudios de piano, los que reemplazó con el estudio del violín y la viola, y sustituyó las actividades físicas por "una abundante lectura" y práctica musical, delineando en gran medida a la carrera musical que desarrollaría a lo largo de su vida (García 1990: 7).

En 1923 ocurrieron varios eventos que marcarían su camino musical. A sus dieciocho años trabajaba contratado como empleado del Banco de Londres y Río de la Plata de Valparaíso y estudiaba composición, armonía, contrapunto y violín de forma particular con distintos músicos en Valparaíso, formándose, en palabras de Fernando García, "febrilmente como músico profesional" (García 1990: 15). Decidió renunciar a su trabajo del banco para dedicarse a la música a tiempo completo y formar su propia orquesta de jazz y le escribió al compositor y dentista Alfonso Leng, quien en ese entonces oficiaba como su mentor, para pedirle consejo. Ante ello, Leng le escribió varias cartas intentando convencerlo de que cambiara de opinión y no se dedicara de manera profesional a la música. En su séptima carta, Leng escribió:

La vida de los artistas es la vida de las preocupaciones por falta de dinero, preocupaciones que absorben y quitan tiempo y entusiasmo. ¿No se podría hacer un arte más puro, menos exhibicionista, es cierto, pero más íntimo viviendo de otro trabajo que no sea de orden musical, para no tener que subordinar jamás el arte ni prostituirlo para poder vivir de él? A mí me parece bien, que si el banco no le paga, se salga, pero ¿no tiene Ud. ahí un porvenir? ¿No habrá otro empleo que le permita a Ud. vivir tranquilo y estudiar su arte sin que tenga que tocar en orquestas shymmys o tangos? [...] Yo le tengo horror a tener que vivir del arte y tener que hacer con él lo que más me repugna para poder vivir. Piénselo bien, yo creo que se puede hacer un arte más puro, no teniendo que verse obligado a comer de ese arte, dándole gusto a la vulgaridad (Leng 1923).

De esta carta desprendemos dos asuntos importantes. Primero, el evidente menosprecio de Leng hacia la música popular, por considerarla poco artística. Segundo, su actitud despectiva hacia los músicos que vivían de la música y, en particular, de la música popular, argumentando que el "subordinar" el arte a motivos económicos, es sinónimo de "prostituirlo" (Leng 1923). A lo que Garrido se estaba enfrentando aquí era a una discusión respecto de si era posible entender la música como trabajo o solo como arte, y que, además, la música a la que él quería dedicarse, el jazz y otras músicas bailables, no eran "arte" sino que algo vulgar (Leng 1923).

Pese a ello, Garrido optó por su carrera musical, combinando distintos trabajos en géneros musicales diversos. En agosto, su obra "Tonada" fue estrenada en el Teatro Victoria de Valparaíso por el pianista Armando Palacios, quien ya tenía una importante trayectoria internacional (García 1990: 15). Al año siguiente, en 1924, formó y dirigió su 
Royal Jazz Orchestra, contribuyendo con ella al entonces incipiente desarrollo del jazz en Chile (Ver Figura 1). También tocó en la orquesta de su hermano Juan Santiago en locales nocturnos en Valparaíso, como Los Baños del Parque, interpretando charlestons, shimmies y maxixes (Menanteau 2006: 29; García 1990: 17, 21). Un año después, en 1925, se asoció a la Sociedad Musical de Socorros Mutuos de Valparaíso, al igual que su profesora de violín Emma Spuhr y varios de los músicos con los que tocaba, como el pianista Carlos Romero, el baterista Bruno Schaub y el violinista Ángel Ceruti (SMSMV). Esta Sociedad la habían fundado cuarenta músicos en diciembre de 1893, con el objetivo de administrar un fondo autónomo y proveer ayuda mutua entre los miembros, ofreciendo, por ejemplo, servicios de médico y farmacia, una pensión cuando algún músico no pudiera trabajar por enfermedad, el pago del funeral de los socios fallecidos, entre otras cosas (Karmy 2017: 545; Karmy y Molina 2018: 60-61).

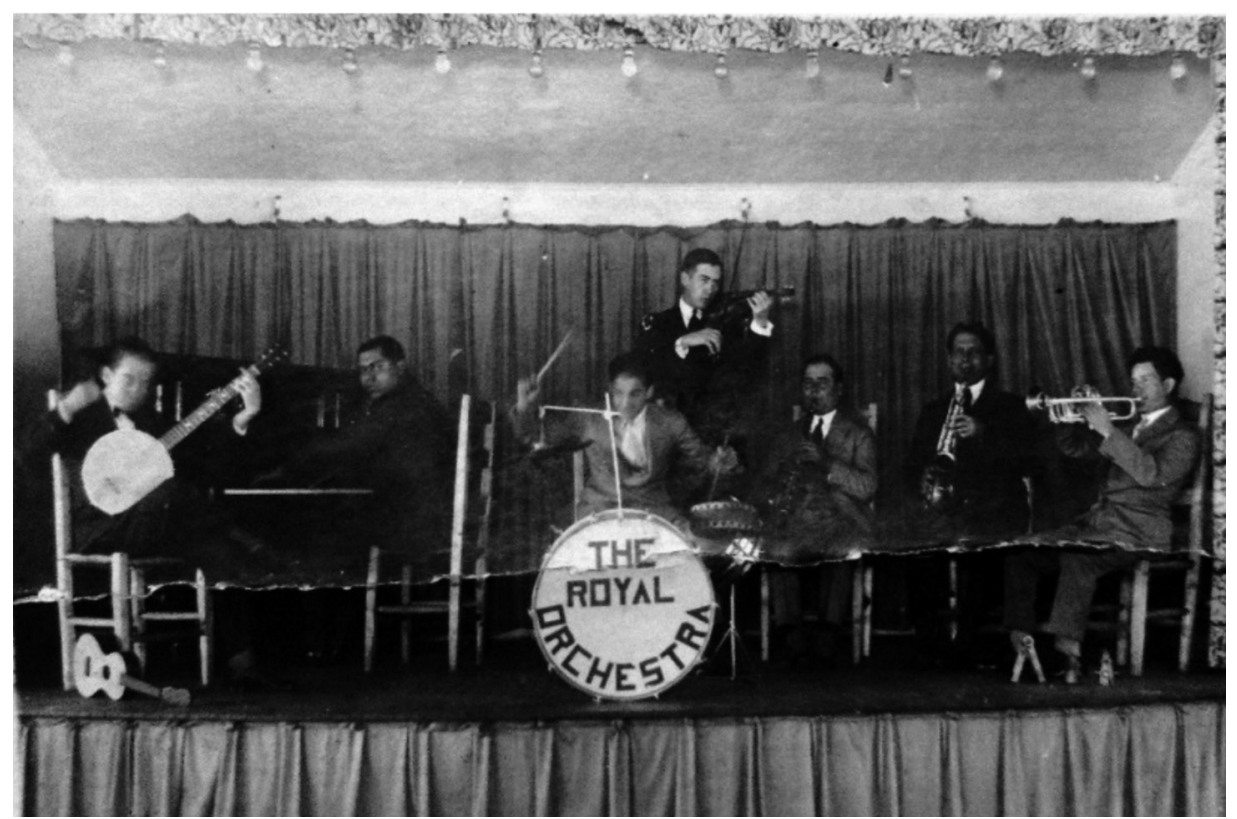

Figura 1: Pablo Garrido (violín) y su Royal Orchestra: banjo, piano, batería, violín triangular, clarinete, saxofón y trompeta, Valparaíso 1925 (FPG, CEDIM).

Pero Garrido no solo estaba interesado en la música popular, ya que desde muy joven se volcó a la música de vanguardia. Organizó el primer concierto de música futurista en Chile, que tuvo lugar el 21 de enero de 1925 en el salón Steinway de Valparaíso, donde compositores de Valparaíso y Santiago interpretaron piezas nacionales para piano (García 1990: 19). Aquí Garrido presentó algunas de sus obras, como "Ascensor", "Una semana y un choapino", "Raid en góndola" y "Fábrica".

Con esto, vemos a Garrido como un activo participante de las escenas musicales docta, de vanguardia y de jazz, lo que le suma complejidad a su perfil comúnmente asociado solo a la música popular por su aporte al jazz y al folclor. Como se ha dicho, su experiencia como compositor vanguardista y de piezas doctas que crearía más adelante, solo recientemente 
ha comenzado a ser estudiada en profundidad (Fugellie 2019). Es importante hacer esta acotación, ya que se suele considerar que el diagnóstico que hizo Garrido acerca de la situación de la música y los músicos chilenos, así como las organizaciones gremiales de músicos que emergieron en este periodo, se enmarcaban solo en la escena de la música popular (González y Rolle 2005: 266-268; Torres 2017). Sin embargo, como veremos a lo largo de este artículo, Garrido incluyó a músicos de todos los géneros tanto en su análisis como en sus propuestas, porque su diagnóstico tenía más que ver con la naturaleza del trabajo musical que de un género en particular.

En la segunda mitad de los años veinte, Garrido trabajó en orquestas de teatros en Santiago, Chuquicamata y Antofagasta, ganó concursos con sus foxtrots, formó conjuntos de cuerda, compuso música para ballet ${ }^{4}$ (García 1990: 22-25) y la célebre Jazz Window para piano y saxo alto, que se considera como "la primera obra docta nacional escrita para este último instrumento" (Menanteau 2006: 29). En las mencionadas ciudades del norte realizó "una notable labor en áreas de la música y la cultura en general" (García 1990: 23), fundó la revista de literatura y poesía Acronal, realizó conferencias musicales y dirigió la orquesta de cine del Teatro Latorre (García 1990: 22-23). Con la llegada de las tecnologías del cine sonoro a Antofagasta a comienzos de la década del treinta despidieron a su orquesta y Garrido quedó cesante. Buscando nuevas oportunidades laborales, se unió a la orquesta de variedades de su hermano Juan Santiago, recorriendo Perú, Ecuador, Colombia y Panamá, donde la orquesta finalmente se disolvió.

En febrero de 1932 Garrido viajó a Europa, donde compartió con artistas como Pablo Picasso, el poeta Vicente Huidobro y el compositor Acario Cotapos; estudió etnomusicología con Andreas Liess en la Sorbonne de París, sin dejar de componer ni de escribir para los diarios chilenos (García 1990: 30-36, 44). En su estadía en París, sufrió las consecuencias de la crisis económica de 1929; frente a ello, la Federación de Músicos de Francia prohibió a extranjeros trabajar sin autorización. Al no encontrar trabajo, debió regresar a Valparaíso en julio de 1932, donde comenzaría una nueva etapa en su vida profesional (García 1990: 44). Fue elegido secretario del directorio del recién formado Sindicato Profesional de Músicos de Valparaíso ese mismo mes (González 1983: 40). Este sindicato, si bien obtuvo su personalidad jurídica en 1932, venía formándose desde 1928 cuando el directorio de la Sociedad Musical de Socorros Mutuos de Valparaíso escribió el primer borrador de los estatutos del Sindicato de Profesores de Música de la Provincia de Aconcagua que, en 1931, pasaría a ser el Sindicato Profesional de Músicos de Valparaíso (Karmy 2019: 143-151).

Probablemente su experiencia en Francia, junto con las impresiones que le compartía su hermano Juan Santiago acerca del Sindicato de Músicos de México, dieron forma a sus ideas respecto del rol que los sindicatos podían cumplir en la protección de los músicos chilenos $^{5}$. Además, sus viajes a lo largo del país y al extranjero lo ayudaron a conocer en profundidad la situación de músicos en distintas condiciones, dedicados a diversos repertorios y estilos musicales.

Los datos recabados en esta investigación sugieren que su trabajo periodístico y etnomusicológico, junto con su posición política, fueron cruciales para sensibilizarse por los derechos laborales de los músicos. Su trabajo periodístico y etnomusicológico lo llevó a viajar por distintas ciudades del mundo, conociendo de primera fuente la realidad de músicos trabajando en condiciones muy diversas; a muchos los entrevistó para sus notas

4 Hojas de otoño es la primera composición de Garrido para ballet, aunque no figura en su catálogo de obras (García 1990: 24).

5 Ver cartas de Juan Santiago Garrido a Pablo Garrido del 2 de noviembre 1932 y del 30 de mayo 1933 (Carpeta Correspondencia Familiar de Pablo Garrido, FPG, CEDIM). 
periodísticas. Su posición política se fue haciendo cada vez más explícita, en línea con el crecimiento institucional de la izquierda chilena, lo que vemos reflejado en sus escritos, sus composiciones musicales y su participación en organizaciones de carácter revolucionario. Por ejemplo, entre 1932 y 1933 compuso obras con contenido revolucionario como Recabarren ${ }^{6}$ (con texto de Max Miranda), Ruge un violento tiroteo (con texto de Julio Walton); Canto a Anabalón basado en el poema Los pequeños proletarios de Carlos Pardo; Elegía a Lenin y Serenata roja (García 1990: 5, 50). Participó también en organizaciones políticas, como la Federación de Artistas Revolucionarios Proletarios de Valparaíso y la Federación Obrera de Chile, sede Valparaíso, de la que fue secretario en 1933 (García 1990: 62; González 1983: 40). En 1937, luego de la llegada de los exiliados republicanos de la guerra civil española, Garrido se sumó al Comité Pro Paz y Ayuda Centro Republicano Español, en Santiago (García 1990: p. 62; Nállim 2019: 5). Entre 1937 y 1938 fue secretario de la Alianza de Intelectuales de Chile (AIDC) que presidía Pablo Neruda (García 1990: 62) y reunía un "amplio espectro de políticos e intelectuales, desde comunistas a liberales", representando cultural e intelectualmente al Frente Popular y la centro-izquierda chilena (Nállim 2019: 5). La relevancia de esto último la analizo más adelante en el artículo, pero por ahora es importante tener en mente que Garrido, al ser una figura clave tanto en los sindicatos de músicos como en la AIDC, muy probablemente lideró y facilitó acuerdos y colaboraciones entre ambas organizaciones.

Las actividades que desarrolló Garrido como defensor de los derechos de los músicos, las podemos resumir en las siguientes: en 1925 se afilió a la Sociedad Musical de Socorros Mutuos de Valparaíso; en 1932 fue electo secretario del Sindicato Profesional de Músicos de Valparaíso, en 1936 presidente, cargos que ocupó por un año. Luego, en 1939, fue elegido presidente del Sindicato Profesional Orquestal (SIPO), ubicado en Santiago. Al mismo tiempo, participó en las organizaciones de carácter revolucionario ya nombradas. Y, por último, cuando se desempeñaba como presidente del SIPO organizó el Primer Congreso de Músicos de Chile, que tuvo lugar en julio de 1940, del que surgiría la Federación de Músicos de Chile. Este último evento marcó un hito en la organización musical en Chile, con el que no solo se buscaba la creación de sindicatos de músicos a lo largo del país sino también la mejora de las condiciones laborales de los músicos.

\section{PREPARACIÓN DEL PRIMER CONGRESO DE MÚSICOS}

$\mathrm{Al}$ analizar los artículos que Pablo Garrido publicó en la prensa, evidenciamos un trabajo de preparación durante gran parte de la década del treinta para este Congreso. La gran mayoría de estos artículos fueron publicados en la prensa nacional de circulación masiva, como el diario Las Últimas Noticias y las publicaciones regionales del diario El Mercurio. En el primero, Garrido fue columnista regular con sus propias secciones: "Crónicas de Pablo Garrido" e "Impresiones de viaje". En la primera incluía notas, entrevistas y crítica musical, mientras que en la segunda, apuntes acerca de sus viajes tanto nacionales como internacionales. Sus artículos de contenido abiertamente político fueron publicados en medios especializados, como las revistas de vanguardia Arte y Multitud, o el periódico del Frente Popular, El Popular de Antofagasta.

6 Luis Emilio Recabarren (1876-1924) es considerado uno de los líderes más importantes del movimiento obrero chileno. Él fundó el Partido Obrero Socialista en 1912, que en 1922 se transformaría en el Partido Comunista de Chile, luego de unirse a la Tercera Internacional (Witker 1977: 78-90; DeShazo 1983: xxv, 135). 
De este modo, mi argumento es que Garrido preparó a sus colegas músicos, al público, a las autoridades y también a los empleadores de los músicos, dándoles a conocer mediante estos escritos sus principales problemas, necesidades y demandas. Estos artículos fueron publicados en medios escritos de distintas ciudades del país, entre 1932 y 1940. En ellos Garrido abordó un sinfín de temas, incluyendo viajes, conciertos, su admiración por los automóviles y aviones y curiosidades acerca de perros ilustres. Por razones metodológicas, me centré solamente en aquellos textos en los que discutió temas que posteriormente se tratarían en el Primer Congreso de Músicos, como la legislación existente y su impacto en el trabajo musical, la necesidad de difundir en mayor medida la música chilena y la formación y fortalecimiento de sindicatos de músicos. Considero que estos artículos sirvieron para poner sobre la mesa la discusión acerca de las condiciones laborales de los músicos, concientizando a sus pares a sindicalizarse en miras de lo que sería el Congreso de 1940, y a visibilizar su estatus de trabajadores. Garrido planteaba que era necesario reconocer a los músicos no solo como artistas, sino también como trabajadores, ya que solo así la actividad musical alcanzaría mayor reconocimiento social y los músicos podrían mejorar sus condiciones laborales. Sin embargo, no definía a los músicos como trabajadores comunes y corrientes, ya que sus actividades eran de carácter intelectual y artístico, lo que es evidente en una de sus publicaciones tempranas:

El "Músico" parece estar relegado a un plano inferior [...] Compréndase que un músico labora tal como un médico, un abogado o un oficinista - y considérese también que sus labores son de índole artística e intelectual (Garrido 1930, énfasis en original).

Esta definición se acerca bastante a cómo los estudios del trabajo musical han entendido a los músicos, como "un tipo particular de trabajadores, buscando empleo pagado en música” (Williamson y Cloonan 2016: 8). El rol que cumplió Garrido en este respecto es clave para entender la identificación de los músicos en tanto trabajadores y artistas. Él fue uno de los pocos que en Chile analizó públicamente la situación laboral de los músicos y relacionó su precario estado a las consecuencias del cine sonoro, la radiodifusión, junto con la legislación que protegía ciertos trabajos y dejaba otros al alero del autocuidado y la ayuda mutua. Aunque aquí analizaremos exclusivamente sus artículos periodísticos, es importante mencionar que Garrido no se quedó solo en las palabras. Lideró una campaña por la defensa y protección de la música y los músicos chilenos, participando de forma activa en organizaciones gremiales, además de plasmar y difundir su diagnóstico y propuestas en diarios locales.

Garrido analizó el impacto de la legislación vigente en el trabajo musical basado en cuatro preocupaciones principales. La primera era que se aprobara el proyecto de ley que crearía más adelante el Instituto de Extensión Musical (IEM), el que emergió en octubre de 1940, patrocinando la formación de instituciones musicales de carácter nacional, como una orquesta sinfónica, coro y ballet. Ya en 1939 había instado a los parlamentarios a aprobar este proyecto de ley, esperando que la creación de distintas instituciones musicales de carácter nacional significaría "la solución de nuestros desvelos" (Garrido 1939a). Creía que estas contribuirían a mejorar las condiciones laborales de los músicos y que los sindicatos tendrían participación en el diseño y ejecución del IEM.

La segunda preocupación era la legislación sobre propiedad intelectual y derecho de autor, la que protegía en mayor medida a los compositores que a los intérpretes. Aunque la Ley 345 de 1925 garantizaba de por vida su propiedad intelectual, no regulaba asuntos de reproducción ni ejecución, dejando también a los compositores en cierto grado de desprotección, por lo que Garrido denunció que comúnmente estos vendían su trabajo "por una ridícula suma" mientras los editores eran quienes hacían "el negocio" (Garrido 
1928). También esta ley definía el derecho a "distribuir, vender o aprovechar con fin de lucro una obra de la inteligencia”, pero no establecía qué entidad era la responsable de supervisar y distribuir esos derechos (Diario Oficial 1925). Solo diez años después de la promulgación de la ley de propiedad intelectual se implementó el primer organismo que buscaba salvaguardar, recaudar y repartir los derechos de ejecución pública: el Pequeño Derecho de Autor. Sin embargo, los problemas de derechos siguieron sin resolverse por completo, y se incluyó la necesidad de modificar esta legislación en el temario del Congreso de Músicos de 1940.

Una tercera preocupación era la ley de previsión social y empleados particulares que regulaba el sistema de administración y previsión de seguridad social de los trabajadores. Ni la caja de ahorro ni la ley de seguro social obligatorio -establecida en 1924 para empleados y profesionales - funcionaban para la gran mayoría de los músicos, ya que estas no incluían a trabajadores independientes. Garrido consideraba que por la falta de una ley de previsión social adecuada "el profesional de la música se debate en las condiciones más adversas" y mejorar esta situación significaba "darle al músico las garantías a que es acreedor todo ciudadano", es decir, un fondo de seguridad social al que podía acceder cualquier otro trabajador (Garrido 1939d). Insistió en la necesidad de regular la protección social de los músicos, acorde a sus condiciones laborales específicas, estableciendo que uno de los objetivos centrales del Congreso sería discutir "los problemas de leyes de previsión social" y así evitar las comunes "colectas para ayudar a compañeros enfermos, en desgracia o a las familias de fallecidos" (Garrido 1939d).

Por último, escribió acerca de la necesidad de crear una ley que protegiera a los músicos chilenos de la competencia que generaban los músicos extranjeros. Aunque este no era un tema nuevo, surgió como un problema después de la cesantía de inicios de los años treinta. Para ejemplificar cómo otros países abordaron este problema entrevistó a Samuel Contreras, un músico chileno trabajando en Buenos Aires, quien destacó los logros de la Asociación General de Músicos de Argentina (AGMA) (Garrido 1939h). Siguiendo ese modelo, el SIPO, bajo la presidencia de Garrido, diseñó un plan en julio de 1939 que incluía la creación del carnet profesional del músico, el que permitiría trabajar a nacionales y a extranjeros con al menos un año de residencia en Chile (Garrido 1939b).

Aunque no todos eran igualmente importantes para los músicos y sus organizaciones, Garrido trató estos cuatro temas a lo largo de la década. Más adelante estos cuatro puntos fueron incluidos en el temario del Congreso de Músicos de 1940, lo que indica que los músicos sindicalizados los reconocieron como temas relevantes.

Los escritos de Garrido estuvieron centrados en la promoción de organizaciones gremiales de músicos, especialmente sindicatos, enfatizando en la necesidad de crear, mantener y fortalecer estas organizaciones con el fin de preparar el camino hacia el primer Congreso de Músicos de Chile y crear la Federación Nacional de Músicos. Esta última, planteaba, buscaría la protección de la música y los músicos chilenos junto con el mejoramiento de la educación musical en todos los niveles (Garrido 1940a). Escribió también acerca de los principales temas que se discutirían en este congreso, creando conciencia respecto de la importancia de esta instancia gremial tanto para los trabajadores de la música como para el público general.

$\mathrm{Al}$ interior del SIPO se creó en 1938 la Sociedad de Compositores con setenta miembros, entre ellos Garrido, quien explicó que esta buscaba editar, publicar y grabar música nacional para ayudar a difundirla en distintos medios, distribuyendo "sus composiciones gratuitamente a los artistas y cantantes", con el fin de promover su ejecución y circulación (Garrido 1940b). Es importante mencionar que esta Sociedad reunía a compositores que no tenían representación en ninguna de las sociedades de compositores ya existentes, como la Asociación Nacional de Conciertos Sinfónicos, la Asociación Nacional de Compositores y la Sociedad de Compositores Chilenos. Estas reunían solo a compositores doctos y eran 
indiferentes "hacia los que escriben obritas de carácter popular o bailable", que eran justamente quienes formaron esta Sociedad (Garrido 1940b).

Analizando los principales problemas de los músicos, en julio de 1939, el SIPO decidió enfrentar estos problemas con acciones específicas. Diagnosticaron que los músicos necesitaban actividades integrales, entendiendo la importancia de la educación y el bienestar entre los miembros, por lo que crearon una sección deportiva para organizar competencias, excursiones y campeonatos de fútbol. El objetivo era ofrecerles un pasatiempo saludable a los músicos y ayudar a estrechar lazos entre los socios, un elemento clave para el fortalecimiento del Sindicato (Garrido 1939b). El SIPO organizó también un festival de orquestas, el cual Garrido consideró que demostró la calidad de las agrupaciones que participaron y sobre todo la colaboración de los miembros para hacer este evento posible (Garrido 1939c).

Aún más importante fue el petitorio que redactó el SIPO en noviembre de 1939 solicitando un día de descanso para el próximo Día de la Música. Las organizaciones gremiales de músicos tenían una larga tradición de celebrar el Día de la Música en honor a Santa Cecilia cada noviembre (Karmy 2019: 131-134). Ahora, este día marcaría un hito estableciendo un día de descanso semanal que los empleadores debían respetar (ver Figura 2). Garrido publicó un artículo donde pedía la colaboración de los locatarios, quienes, una vez a la semana, debían abstenerse de pedir a los músicos que fueran a trabajar (Garrido

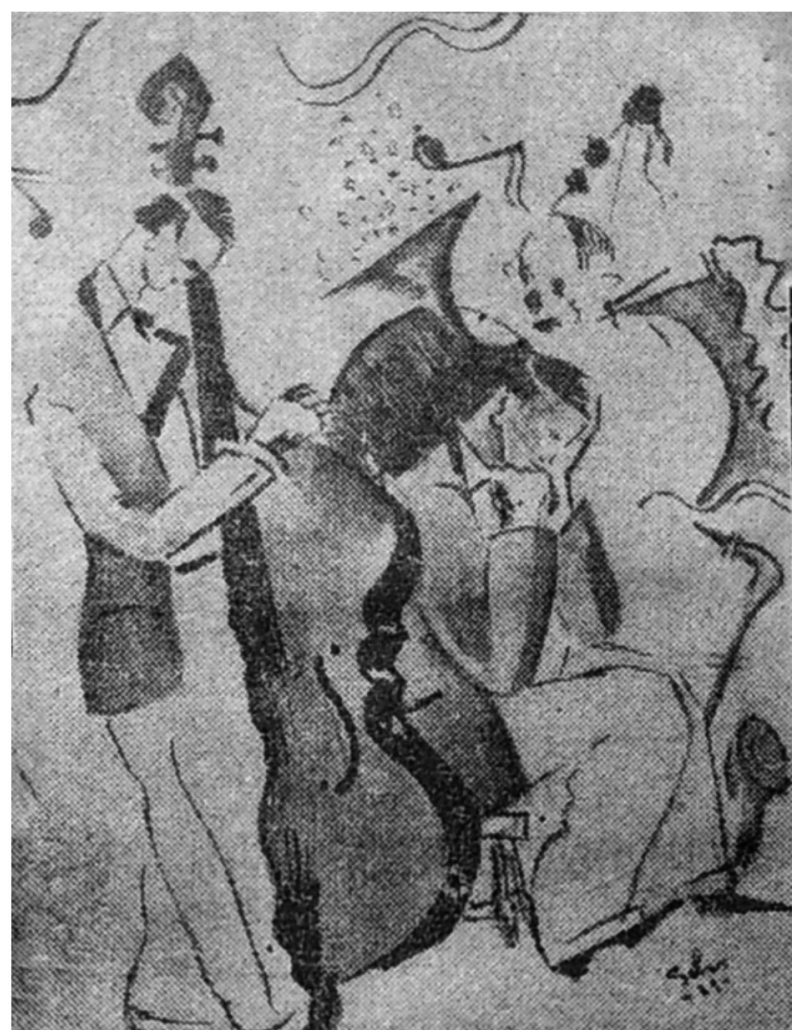

Figura 2: Dibujo de Jorge Salas para la crónica de Pablo Garrido, "mostrando el agotamiento del músico de orquesta, que trabaja sin Descanso todos los días del año. Piden descanse para el 22 de noviembre, Día de la Música" (Garrido 1939d). 
1939d). Esta petición fue apoyada por diferentes sectores con lo que se puso a prueba el poder del SIPO, ya que este sancionó a "algunos elementos que no quisieron respetar el acuerdo entre patrones y músicos” (Garrido 1939e). Esta experiencia serviría como enseñanza para el futuro, esperando que cuando tuviera lugar el Congreso, los músicos estarían "definitivamente unidos todos por el progreso del arte musical de Chile" (Garrido 1939e). Precisamente, con relación a las preparaciones de este Congreso, el compositor e historiador de la música Luis Sandoval celebró la iniciativa planteando que la unidad les daría el poder legal para resolver sus problemas (Garrido 1939g).

En diciembre de 1939 Garrido, aún presidente del SIPO, invitó a los músicos a unirse a un sindicato y prepararse para el Primer Congreso de Músicos de Chile mediante la creación de sindicatos regionales, comités o delegados ${ }^{7}$. El SIPO invitó a diferentes sindicatos y comités -cuando no pudieran reunirse más de veinticinco músicos- de distintos lugares del país a participar en el Congreso (Garrido 1939f).

Un mes antes del Congreso, Garrido escribió que después de varios meses de preparaciones para esta "justa gremial artística", cerca de "5.000 músicos chilenos" estaban "pendientes de su próximo congreso" (Garrido 1940a). Enfatizando las precarias y diversas condiciones laborales de los músicos, consideró como "profesionales de la música" a todos quienes trabajaran en "bandas, de ópera, de orquesta sinfónica, de conjuntos de cámara o de orquestas bailables" (Garrido 1940a). Destacó que los sindicatos de músicos de Valparaíso, Rancagua, Concepción, Lota y Magallanes ya apoyaban al Congreso, representando las principales ciudades a lo largo del país. Garrido esperaba que más sindicatos participaran para contribuir a mejorar las condiciones de los músicos profesionales de Chile (Garrido 1939f). Pero al mismo tiempo, clarificó que esta reunión gremial no era solo para músicos sindicalizados sino también "cientos de maestros dedicados a la enseñanza privada, los aficionados en general y muy en particular todos los artistas e intelectuales del país, quienes ven en este movimiento, el nacimiento de una conciencia nueva" (Garrido 1940a).

Esto significaba que los músicos ahora tenían conciencia de su situación y veían en el sindicalismo la mejor forma para enfrentar sus problemas. Garrido pensó que sus problemas se resolverían pronto, gracias al poder de sus sindicatos y las conclusiones del Congreso y expresó su optimismo escribiendo que, después de mucho tiempo, el SIPO había "despertado brioso, laborando con singular tesón” (Garrido 1939d). El creciente poder de los sindicatos se percibía en las preparaciones del Congreso, que Garrido entendió como reflejo de unidad y el camino a seguir para lograr sus objetivos (Garrido 1939f). Esperaba que los resultados del Congreso beneficiaran a todos quienes trabajaban como músicos y al público en general, destacando la relevancia social de la música y la profesión musical, ya que estas contribuirían a "la cultura misma del pueblo chileno" (Garrido 1940a). Este optimismo, sin embargo, se ensombrecía por el escaso aporte de las instituciones públicas, por lo que sería la organización misma de los músicos la que les garantizaría sus derechos (Garrido 1939f).

En su "Manifiesto a los músicos de Chile" que circuló en diciembre de 1939 en forma de panfleto, Garrido resumió los principales temas que se discutirían en el Congreso: la aplicación de la ley de empleados particulares "o [la promulgación de] una ley especial de previsión social"; el uso del carnet profesional "como defensa del músico chileno"; y la “capacitación obligatoria, para elevar el nivel artístico y cultural del profesorado” (Garrido

7 Los estatutos del Congreso de Músicos estipulaban que los delegados "deberán ser elegidos democráticamente en Asamblea" y que "deberán ser nombrados en proporción al número de socios que tenga la organización” (SIPO 1940b: 9). Por ejemplo, los músicos de las ciudades de Coquimbo y La Serena fueron representados por el delegado Emilio Contreras, electo por los respectivos comités (SIPO 1940a: 3). 
1939f). Ya en julio el SIPO había diseñado un plan de capacitación musical para sus propios miembros que contemplaba la creación de orquestas propias, talleres de dirección y cursos de actualización impartidos por el Conservatorio Nacional de Música (Garrido 1939b). Pero también los músicos profesionales buscaban aportar a mejorar la educación musical en el país, tratando los problemas pedagógicos de la educación musical en las escuelas básicas y secundarias y creando orquestas y escuelas populares de música "para hacer llegar la cultura al pueblo", es decir, a la clase trabajadora (SIPO 1940b: 21). Con eso, planteaban, podían contribuir a un país "donde el arte musical asuma la plenitud de su rol social" sumándose al proyecto del Frente Popular (SIPO 1940b: 21). Todas estas propuestas fueron desarrolladas en el "Manifiesto a los músicos del país" adjunto a la convocatoria al Congreso, que firmó el comité organizador integrado por miembros de la Orquesta Sinfónica Nacional, el Club Musical, el Sindicato de Músicos de Bandas y el SIPO ${ }^{8}$, y cuyo secretario general era Garrido (SIPO 1940b: 19-24). Se definió al músico profesional como un "trabajador intelectual" a quien se le instó a mantener una "férrea unión" con sus colegas mediante su participación en el próximo Congreso (Garrido 1939f).

Además de la creación de nuevos sindicatos y de fortalecer los ya existentes, el objetivo era unir estas organizaciones a nivel nacional mediante la creación de la Federación de Músicos de Chile. Haciendo hincapié en las actividades y logros del SIPO y otros sindicatos durante esta década, Garrido proyectó que, con el próximo Congreso, los "músicos de todo Chile [...] van a cumplir totalmente con sus firmes propósitos” (Garrido 1940a).

\section{EL PRIMER CONGRESO DE MÚSICOS DE CHILE}

Este Congreso se realizó entre el 1 y el 5 de julio de 1940 en el Teatro Municipal de Santiago, donde participaron diversas organizaciones, representando a cinco mil músicos a lo largo del país (Garrido 1940c; SIPO 1940a). Es interesante constatar que esta actividad ubicó a los músicos entre el movimiento obrero y el movimiento artístico, develando la complejidad del trabajo musical y su doble faz en tanto trabajo y arte. Por ejemplo, en el "Manifiesto a los músicos del país", no solo se reivindicó el movimiento obrero, sino que también se planteó que, para alcanzar sus logros, los músicos debían seguir el mismo camino:

Si observamos atentamente el progreso orgánico de los trabajadores, su unidad indestructible y su espíritu de lucha, si vemos, cómo día a día, logran mayores conquistas a través de esa misma unidad, y cómo la potente Confederación de Trabajadores de Chile hace respetar los derechos ciudadanos y apoya los movimientos de reivindicaciones, logramos descubrir un camino que debemos seguir necesariamente: la UNIDAD. (SIPO 1940b: 19-20, énfasis en original).

8 El comité organizador del Congreso estaba compuesto de la siguiente forma por integrantes del SIPO, el Sindicato de Músicos de Banda (SMB), la Orquesta Sinfónica Nacional (OSN) y el Club Musical (CM):

Secretario general: Pablo Garrido.

Secretario de actas: Ernesto Garrido (OSN).

Prosecretario: Álvaro Pinto (CM).

Secretario de prensa y correspondencia: Francisco Piñats (CM).

Asesores: R. Hermosilla (SIPO) y Luis Cavieres (SMB).

Secretario de agitación y propaganda: Venancio Yáñez (SMB).

Asesores: Filiberto Baronti (OSN), Luis Astorga (SIPO) y Alberto L. Almarza (CM).

Secretario de tesorería: R. Neira (SIPO); asesor: Juan Amigo (OSN).

Comisión receptora de poderes y orden del día: Sofanor Navarrete (SIPO), Juan Amigo (OSN), Luis Novoa (SMB), Álvaro Pinto (SMB) y Enrique Maura (SIPO) (SIPO 1940b: 24). 
$\mathrm{Al}$ reunirse en Congreso, los músicos sindicalizados seguían el modelo del movimiento obrero, haciéndose representar por su órgano central, la Confederación de Trabajadores de Chile (CTCh). El llamado a unirse a la CTCh fue explícito, lo que implicaba entender abiertamente a los músicos como trabajadores. Pero al mismo tiempo, la particularidad del trabajo musical se enfatizó en la necesidad de analizar la legislación relativa a pensiones y bienestar social, porque esta no era aplicable a los trabajadores independientes, como los músicos. Se convocó a "todos los músicos profesionales de Chile, sea cual fuere el aspecto de la profesión en que se desempeñen: pequeños conjuntos, orquestas de cámara, orquestas sinfónicas, bandas civiles, etc." a discutir acerca de su situación como un tipo particular de trabajadores (SIPO, 1940b: 19). Al incluir también a intérpretes de la Orquesta Sinfónica Nacional y compositores ligados al Conservatorio, el Congreso se posicionó también como un evento artístico, con presentaciones musicales a cargo de dichas instituciones, ayudándole a ganar reconocimiento social e intelectual.

De este modo, los músicos se reunieron en un congreso en el que participaron los sindicatos de músicos, gremios y comités de todas las regiones del país, "organizaciones orquestales, conjuntos sinfónicos, músicos de ópera, músicos de bandas civiles, etc.," y la "participación de representantes de la C.T.CH." (SIPO 1940b: 7). Además, el lenguaje que se usó en la invitación y en el programa impreso era abiertamente combativo, siguiendo el modelo de las luchas reivindicativas del movimiento obrero. La invitación explicitaba el objetivo de constituir una Federación Nacional de Músicos y de establecer un vínculo con el movimiento de trabajadores, uniéndose a la CTCh, cuya influencia era significativa por tener representación en el gobierno (SIPO 1940b: 4-5). La invitación decía que los "músicos chilenos han vivido, hasta ahora, al margen de los beneficios de que debe gozar todo ciudadano, abandonados de todas las leyes sociales" (SIPO 1940b: 3). Al comparar a los músicos con trabajadores de otros oficios que sí podían recurrir al sistema de previsión social, relevaba la situación de precariedad en que trabajaban. Ante esto, la Federación vendría a "resolver los problemas más fundamentales del gremio" (SIPO 1940b: 3). Finalmente, las demandas que surgirían en el Congreso serían presentadas a ambas cámaras del Parlamento. De estas, la más importante era promulgar una ley de previsión social acorde al trabajo de los músicos y que "asegure su vejez" (SIPO 1940b: 21).

Durante los cuatro días del Congreso hubo discusiones organizadas en cuatro sesiones plenarias de tres horas cada una y, en el último día, una sesión de una hora con las resoluciones (SIPO 1940b: 18). En la primera sesión se eligió a la mesa directiva del Congreso, integrada por un secretario general, tres presidentes, tres secretarios de actas, dos secretarios de prensa, quienes rotaron para dirigir esta reunión gremial (SIPO 1940b: 10). Hubo también discursos y espectáculos artísticos que se transmitieron en vivo por Radio Cooperativa Vitalicia. Entre los discursos que se realizaron se encuentran el saludo inaugural a las delegaciones por Juan Amigo, miembro del comité preparatorio, una charla titulada "Significación del Primer Congreso de Músicos de Chile" presentada por Pablo Garrido, secretario general del Comité Preparatorio; y una alocución del Decano de la Facultad de Bellas Artes, Domingo Santa Cruz (SIPO 1940c). Uno de los espectáculos que se mostró durante el Congreso fue una pieza teatral "basada en la vida real del músico" para educar y crear conciencia entre los asistentes acerca de sus demandas (SIPO 1940b: 17). Recurrir a obras de teatro para informar y educar era una tradición de larga data del movimiento obrero chileno (Garcés 2003:138; Bergquist 1986: 49; Grez 2011: 16-17). Desde mediados del siglo XIX sindicatos obreros y sociedades mutualistas, especialmente de los pueblos salitreros del norte de Chile, pero también a lo largo de las ciudades afectadas por la cuestión social, organizaron grupos teatrales para educar y politizar a los trabajadores y sus familias. Estos promovieron la realización de obras de teatro durante las asambleas 
obreras para concientizar respecto de los problemas que sufría la clase trabajadora e involucrarlos en la lucha (Witker 1977:126-171; Grez 2011: 16-17).

La Orquesta Sinfónica Nacional interpretó piezas musicales dirigidas por Víctor Tevah y Armando Carvajal, así como estudiantes del Conservatorio Nacional de Música, representando a su Centro de Alumnos, hicieron también algunas presentaciones (SIPO 1940a: 4). El repertorio consistió en mayor medida de obras clásicas ${ }^{9}$ que, junto con la importante presencia de músicos de la Sinfónica, desmiente la idea de que Garrido solo buscaba atender a las problemáticas de los músicos populares, señalada al comienzo del artículo. Además, como se observa en el programa del Congreso, Garrido invitó al abogado y compositor docto Domingo Santa Cruz para que participara en su calidad de decano de la Facultad de Bellas Artes de la Universidad de Chile (ver Figura 3). Es importante mencionar aquí que, aunque el Congreso apuntaba a músicos-trabajadores y sindicalizados, no se limitó a

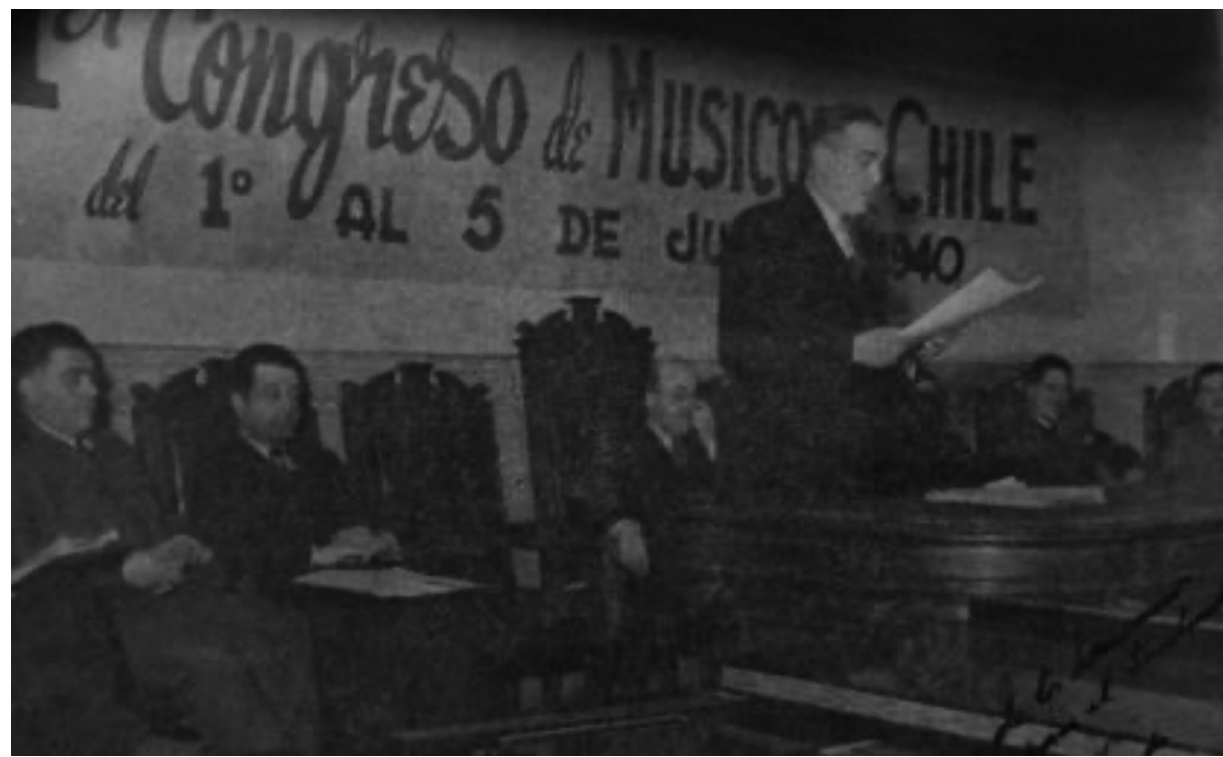

Figura 3: Pablo Garrido presenta su conferencia en el Primer Congreso de Músicos de Chile, 1940. Nótese que detrás de él estaba sentado Domingo Santa Cruz (FPG, CEDIM).

${ }^{9}$ En la inauguración del Congreso se interpretaron las siguientes piezas musicales:

- Obertura Sueño de una noche de verano (F. Mendelssohn) por la Orquesta Sinfónica Nacional, dirigida por Víctor Tevah.

- Fete (C.A. Debussy) por la Orquesta Sinfónica Nacional dirigida por Armando Carvajal.

- "Sueño de Elsa" de la ópera Lohengrin (R. Wagner), por Blanca Hauser (solista) y la Orquesta Sinfónica Nacional, dirigida por Armando Carvajal.

- Balada (F. Chopin) por Herminia Raccagni (piano).

- Concierto en re, primer movimiento (W.A. Mozart) por Tito Dourthé (violín), Amalia Arata (piano), contribución del Centro de Alumnos del Conservatorio Nacional.

- El ruiseñor (F. Liszt) y Estudio op. 10, $n^{\circ} 18$ (F. Chopin) por Carmen Moreno (piano), alumna de perfeccionamiento del Conservatorio Nacional de Música, contribución del Centro de Alumnos del Conservatorio Nacional.

- Canto a la Música, himno oficial de los músicos de Chile (Luis Sandoval Bustamante) por el Conjunto coral del Sindicato Profesional Orquestal, dirigido por Jorge Allende (SIPO 1940c). 
ellos, incluyendo a músicos, como los vinculados al Conservatorio, que no pertenecían a ningún sindicato, ni se identificaban a sí mismos como trabajadores, como el caso de Santa Cruz, quien hiciera ese énfasis en su alocución al señalar que:

[S] in que sea yo precisamente uno de los profesionales a que se alude en el comienzo del 'manifiesto' que convocó a este Congreso, ni la Universidad de Chile una de las entidades gremiales referidas allí mismo, me siento aquí en mi casa y estamos de corazón unidos a esta asamblea a la cual también nos llama en su parte final la convocatoria cuando se refiere a las demás ramas del cultivo de la música en cuyas filas formamos los profesores, los compositores y los ejecutantes no sindicados (Santa Cruz 1940: 2).

\section{TABLA 1: SINDICATOS QUE REPRESENTARON A MÚSICOS PROFESIONALES EN SU PRIMER CONGRESO, 1940, DE NORTE A SUR.}

\begin{tabular}{|c|c|c|c|}
\hline 1 & $\begin{array}{c}\text { Sindicato Profesional Orquestal de } \\
\text { Tarapacá (Iquique) }\end{array}$ & 9 & Sindicato Orquestal de Linares \\
\hline 2 & $\begin{array}{c}\text { Sindicato Profesional Orquestal de } \\
\text { Antofagasta }\end{array}$ & 10 & $\begin{array}{c}\text { Sindicato Profesional Musical de } \\
\text { Concepción }\end{array}$ \\
\hline 3 & Sindicato Orquestal Musical de Chañaral & 11 & Sindicato Profesional de Lota \\
\hline 4 & Sindicato Profesional de Músicos de Ovalle & 12 & Sindicato Profesional de Cautín (Temuco) \\
\hline 5 & $\begin{array}{c}\text { Sindicato Profesional de Músicos de } \\
\text { Valparaíso }\end{array}$ & 13 & Sindicato Profesional Orquestal de Valdivia \\
\hline 6 & Sindicato Profesional Orquestal (Santiago) & 14 & $\begin{array}{c}\text { Sindicato Profesional de Músicos de } \\
\text { Osorno }\end{array}$ \\
\hline 7 & Sindicato de Músicos de Banda (Santiago) & 15 & $\begin{array}{l}\text { Sindicato Profesional Orquestal de } \\
\text { Llanquihue (Puerto Montt) }\end{array}$ \\
\hline 8 & $\begin{array}{c}\text { Sindicato Profesional Orquestal de } \\
\text { O'Higgins (Rancagua) }\end{array}$ & 16 & $\begin{array}{c}\text { Sindicato Profesional Orquestal de } \\
\text { Magallanes (Punta Arenas) }\end{array}$ \\
\hline
\end{tabular}

Fuente: Elaboración propia.

Además del Centro de Alumnos del Conservatorio, la Orquesta Sinfónica Nacional, el Club Musical de Santiago y un delegado que representaba los comités de Coquimbo y La Serena, participaron dieciséis sindicatos de músicos del país (ver Tabla 1). La participación de delegados de distintas zonas del país era esencial para abordar los problemas nacionales tomando en cuenta todas las opiniones, especialmente considerando que la organización sindical tenía un carácter más local que nacional, siguiendo la particular forma geográfica del país -con sindicatos organizados por ciudad o provincia-. Pero para un país centralista, era también crucial que el Congreso considerara las diversas condiciones en que trabajaban los músicos en las distintas regiones (SIPO 1940a:1), tal como ya se había anunciado en diciembre del año anterior: 
Los problemas del músico son fundamentalmente los mismos, pero sus actividades especializadas deben ser miradas con un criterio amplio y total. No es posible aplicar las mismas medidas en la capital como en las lejanas regiones del país. No es posible actuar con criterio centralista, cuando Chile se divide en regiones de fisonomías y actividades muy diversas entre sí (Garrido 1939f).

Reunirse en un congreso nacional, así como organizarse en sindicatos, son prácticas que siguen el modelo del movimiento obrero más que de artistas e intelectuales. Más aún, la CTCh jugó un rol central en aconsejar y guiar a sus participantes para la formación de la Federación Nacional de Músicos que surgió luego del Congreso (Garrido 1941). Pero al mismo tiempo, los espectáculos artísticos que se realizaron en esta instancia enfatizaron la particularidad de los músicos en tanto artistas y no solo trabajadores. Vale la pena mencionar que el SIPO recibió apoyo tanto de la CTCh como de la AIDC, entidades en las que Garrido participó. La primera ayudó al Sindicato en relación con "la defensa de sus intereses gremiales", mientras que la segunda contribuyó con sus vínculos artísticos (Garrido 1939b).

El Congreso tuvo dos consecuencias inmediatas. La primera fue la creación de la Federación Nacional de Músicos, adherida a la CTCh, a la que se unieron los dieciséis sindicatos de músicos que se formaron a lo largo del país desde fines de los años veinte y que se reunieron en este primer congreso (SIPO 1940a: 6, ver Tabla 1). La segunda, fue la realización de un segundo congreso entre el 18 y 21 de noviembre de 1942 en Valparaíso, también liderado por Garrido, quien había sido electo secretario general de la Federación de Músicos, cargo que ocupó hasta 1942 (García 1990: 74; González 1983: 40). Sostengo que el Congreso y, especialmente la labor de concientización que hizo Garrido sobre las condiciones laborales de los músicos, contribuyeron en el largo plazo a reconocer a los músicos profesionales como trabajadores.

\section{CONCLUSIÓN}

En este artículo planteé la necesidad de pensar lo musical desde una perspectiva que incluya lo laboral, ya que permite develar facetas que no conocíamos de los músicos, así como tener una visión integral de su quehacer. El aporte de Pablo Garrido a la música chilena ha sido estudiado de manera más bien aislada que interconectada, destacando la contribución que hizo a distintos géneros musicales en vez que entender su quehacer de manera conjunta. Al entenderlo como un músico-trabajador, buscando vivir de la música, e interesado en mejorar las condiciones laborales del gremio, más allá de un género en particular, advertimos también su aporte a la organización gremial de músicos y la creación de instituciones musicales. Al develar esta faceta, podemos reconocer a Garrido no solo como un artista que aportó al desarrollo de ciertos géneros musicales, sino también como un trabajador liderando un movimiento social para mejorar las condiciones de vida y trabajo de todo el gremio.

Mediante sus artículos periodísticos, analicé la campaña que Garrido lideró para visibilizar la precariedad laboral de los músicos, la falta de difusión de la música chilena y la necesidad de articular sindicatos de músicos a lo largo del país. Su campaña se tradujo en las demandas que se discutieron en el Congreso de Músicos de 1940: la necesidad de instituciones musicales con financiamiento estatal; una nueva legislación para mejorar las condiciones laborales y de seguridad social de los trabajadores de la música, incluyendo propuestas de modificación a la ley de previsión social y a la legislación en propiedad intelectual y derecho de autor. En esta campaña Garrido le asignó una importancia fundamental a la organización gremial para asegurar la unidad entre los músicos, la que se tradujo en la formación de sindicatos y comités locales que se reunieron en dos congresos nacionales en 1940 y 1942. 
Además de las consecuencias directas que tuvo la campaña de Garrido -como la creación de la Federación y la realización del segundo Congreso- argumenté que esta contribuyó a crear conciencia acerca de la situación de la profesión musical. Especialmente, favoreció la identificación de los músicos como trabajadores. Tanto Garrido como los sindicatos no solo reconocieron, sino que también explicitaron el valor artístico de los músicos profesionales, enfatizando igualmente su identificación como trabajadores.

Junto con esto demostré en qué medida los músicos sindicalizados de Valparaíso, Santiago y otras ciudades del país fueron parte tanto del campo musical como del movimiento obrero. Los músicos que se agruparon en sindicatos se encontraron a medio camino entre el movimiento de trabajadores, la escena artística y las industrias musicales, conformando una "aristocracia del trabajo" (Hobsbawm 1984: 355-372), siendo pequeños burgueses con problemas compartidos con el proletariado. Estos problemas tenían relación con la naturaleza independiente y precaria del trabajo musical, materializada, entre otras cosas, por el hecho de que al trabajar en las lógicas de la gig economy, quedaban al margen de la seguridad social que proveía el Estado. Además, que el SIPO, el Congreso y Garrido estuvieran vinculados tanto a una confederación de trabajadores (la CTCh) como a una asociación artística (la AIDC), demuestra la complejidad de la doble faz de los músicos, en tanto artistas y trabajadores. Al relevar esta última faceta, fue posible develar una parte de la historia de la música en Chile que había pasado inadvertida.

\section{ABREVIATURAS}

AGMA: Asociación General de Músicos de Argentina

AIDC: Alianza de Intelectuales de Chile

CEDIM: Centro de Documentación e Investigación Musical de la Facultad de Artes de la Universidad de Chile.

CM: Club Musical

CTCh: Confederación de Trabajadores de Chile

FDS: Fondo Domingo Santa Cruz del CEDIM

FPG: Fondo Pablo Garrido del CEDIM

IEM: Instituto de Extensión Musical

OSN: Orquesta Sinfónica Nacional

SIMUPROVAL: Sindicato de Músicos Profesionales de Valparaíso

SIPO: Sindicato Profesional Orquestal

SMB: Sindicato de Músicos de Banda

SMSMV: Sociedad Musical de Socorros Mutuos de Valparaíso

\section{REFERENCIAS}

Documentos de archivo

Correspondencia Familiar de Pablo Garrido. Ms. (Caja 72 FPG, CEDIM).

García, Fernando

1990 Biografía inédita de Pablo Garrido. Ts. (FPG, CEDIM).

Garrido, Pablo

1928 "Los autores musicales", El Mercurio, Valparaíso, 6 junio. Ts. (Libro 1, FPG, CEDIM).

1930 "Sobre la Sinfónica”, El Mercurio, Antofagasta, mayo. Ts. (Libro 1, FPG, CEDIM). 
1939a "Héroes de la música. La orquesta Sinfónica Nacional. Revelaciones sensacionales”, Las Últimas Noticias, Santiago, 29 de junio. Ts. (Libro 1, FPG, CEDIM).

1939b "Músicos que olvidan la bohemia. Capacitación del músico. Los músicos chilenos quieren trabajar en Chile”, Las Últimas Noticias, Santiago, 20 de julio. Ts. (Libro 1, FPG, CEDIM).

1939c Magníficas son nuestras orquestas”, Las Últimas Noticias, Santiago, 19 de octubre. Ts. (Libro 1, FPG, CEDIM).

1939d “Descanso para el músico. Lo que debe saber el público. Una justa petición de los músicos”. Las Últimas Noticias, Santiago, 10 de noviembre. Ts. (Libro 1, FPG, CEDIM).

1939e “Los músicos chilenos se hacen respetar, dice Lacasia”, Las Últimas Noticias, Santiago, 16 de noviembre. Ts. (Libro 1, FPG, CEDIM).

1939f Manifiesto a los músicos de Chile, diciembre. Ts. (Libro 1, FPG, CEDIM).

1939g "Don Luis Sandoval y el porvenir del músico", Las Últimas Noticias, Santiago, 7 de diciembre. Ts. (Libro 1, FPG, CEDIM).

1939h "Samuel Contreras en B. Aires", Las Últimas Noticias, Santiago, 21 de diciembre. Ts. (Libro 1, FPG, CEDIM).

1940a "Un concurso de músicos. Un acontecimiento histórico en la vida artística chilena", Las Últimas Noticias, Santiago, 6 de junio. Ts. (Libro 1, FPG, CEDIM).

1940b "Cruzada de compositores chilenos", Las Últimas Noticias, Santiago, 20 de junio. Ts. (Libro 1, FPG, CEDIM).

1940c "Tendremos música chilena”, Las Últimas Noticias, Santiago, 21 de noviembre. Ts. (Libro 1, FPG, CEDIM).

1941 "Es casi paradojal que los músicos chilenos ignoren la riqueza de su propia música”, El Popular, Antofagasta, 10 de julio. Ts. (Libro 1, FPG, CEDIM)

Leng, Alfonso

1923 "Carta a Pablo Garrido”. Ms. (Caja 50, carta 7, FPG, CEDIM).

Santa Cruz, Domingo

1940 Discurso de Domingo Santa Cruz en Primer Congreso de Músicos de Chile. Ms. (01-E-027. FDS. CEDIM).

SIPO

1940a Boletín Informativo $\mathrm{N}^{\circ} 1$, El Sindical, Santiago, 26 de junio. Ts. (01-E-027. FDS. CEDIM).

1940b Convocatoria y Reglamentos del Primer Congreso de Músicos de Chile a realizarse en Santiago del 1 al 5 de Julio. Ts. (01-E-027. FDS. CEDIM).

1940c Programa de la inauguración del Primer Congreso de Músicos de Chile. Ts (01-E-027. FDS. CEDIM)

SMSMV

Libro de Pagos de la Sociedad Musical de Socorros Mutuos de Valparaíso de 1922 a 1974. Ms. (SIMUPROVAL).

\section{Bibliografía}

Bergquist, Charles

1986 Labor in Latin America: comparative essays on Chile, Argentina, Venezuela, and Colombia. Stanford, Calif: Stanford University Press.

Brook, Orian, Dave O'Brien Y Mark Taylor

2020 Culture is bad for you. Inequality in the cultural and creative industries, Manchester: Manchester University Press. 
Cabello, Pablo

2020 "Historia del Jazz en Valparaíso: Los albores (1920-1934)" [tesis de Magíster en Historia mención en Historia del arte y de la cultura] Pontificia Universidad Católica de Valparaíso.

Cloonan, Martin

2014 "Musicians as Workers: Putting the UK Musicians' Union into Context", MUSICultures, XLI/1, pp.10-29, DOI: https://doi.org/10.1017/S0261143013000093

Cloonan, Martin Y John Williamson

2017. "Introduction", Popular Music and Society, XL/ 5, pp. 493-98, DOI: https://doi.org/10.108 0/03007766.2017.1351117.

DAvid-Guillou, ANGÉle

2009 "Early Musicians' Unions in Britain, France and the United States: On the Possibilities and Impossibilities of Transnational Militant Transfers in an International Industry", Labour History Review, LXXIV/3, pp.88-304, DOI: 10.1179/096156509x12513818419655.

Deshazo, Peter

1983 Urban workers and labor unions in Chile, 1902-1927. Madison, Wis: University of Wisconsin Press.

Donoso, Karen y Carolina Tapia

2017 "(De)construyendo el folclor: historia de su conceptualización en la academia universitaria chilena durante el siglo XX”, Mapocho, 82, pp.131-161.

FINNEGAN, RUTH

2007 The Hidden Musicians: music-making in an English town. Middletown, CT: Wesleyan University Press.

Frith, SIMON

2017 “Are workers musicians?”, Popular Music, XXXVI/1, pp.111-115, DOI: 10.1017/ S0261143016000714

Fugellie, Daniela

2019 "Pablo Garrido y la vanguardia dodecafónica" ponencia presentada en Seminario Pablo Garrido y la música del siglo XX, reflexiones en torno a su archivo, Facultad de Artes, Universidad de Chile, Santiago de Chile, agosto.

Garcés, Mario

2003 Crisis social y motines populares en el 1900. Santiago de Chile: LOM.

GonzÁlez, JuAN PABlo

1983 "Cronología epistolar de Pablo Garrido", Revista Musical Chilena, XXXVII/160, pp. 4-46.

González, Juan Pablo y Claudio Rolle

2005 Historia Social de la Música en Chile, 1890-1950. Santiago de Chile: Ediciones Universidad Católica de Chile.

Grez, Sergio

2011 ¿Teatro ácrata o teatro obrero? Chile, 1895-1927”, Estudios Avanzados, 15, pp.9-29.

Gross, Sally Anne Y Musgrave, George.

2020 Can Music Make You Sick? Measuring the Price of Musical Ambition. Londres: University of Westminster Press.

HobsBawm, ERIC

1984 “Artisan or Labour Aristocrat?", The Economic History Review, XXXVII/3, pp.355-372, DOI: $10.2307 / 2597286$

HoOK, LesLie

2015 "Year in a Word: Gig Economy", Financial Times Online. https://www.ft.com/content/ b5a2b122-a41b-11e5-8218-6b8ff73aae15/ 
KARMY, EILEEN

2017 "Musical Mutualism in Valparaiso during the Rise of the Labor Movement (1893-1931)", Popular Music and Society, XL/5, pp. 539-555, DOI: 10.1080/03007766.2017.1348634

2019 "The Path to Trade Unionism: Musical Work in Chile (1893-1940)" [PhD] University of Glasgow.

Karmy, Eileen y Cristian Molina

2018 "Músicos como trabajadores. Estudio de caso de la Sociedad Musical de Socorros Mutuos de Valparaíso (1893-1930)”, Resonancias, XXII/42, pp.53-78, DOI: 10.7764/res.2018.42.4.

KRAFT, JAMES

1995 “Artists as Workers: Musicians and Trade Unionism in America, 1880-1917”, The Musical Quarterly LXXIX/3, pp. 512-43, DOI: http://www.jstor.org/stable/742442.

LOFT, ABRAM

1950 "Musicians' guild and union: A consideration of the evolution of protective organization among musicians" [PhD] Columbia University.

Menanteau, Álvaro

2006 Historia del Jazz en Chile. Santiago de Chile: Ocho Libros.

Miller, Karl Hagstrom

2008 "Working Musicians: Exploring the Rhetorical Ties Between Musical Labour and Leisure", Leisure Studies, XXVII/4, pp.427-441, DOI: 10.1080/02614360802334963.

NÁllim, Jorge

2019 "Culture, Politics and the Cold War: The Sociedad de Escritores de Chile in the 1950s", Journal of Latin American Studies, LI/3, pp. 1-27, DOI: 10.1017/S0022216X18000755.

RAmos, Ignacio

2012 "Políticas del folklore. Representaciones de la tradición y lo popular. Militancia y política cultural en Violeta Parra y Atahualpa Yupanqui”. [tesis de Magíster en Estudios Latinoamericanos]. Universidad de Chile.

Silva, Manuel Deniz

2017 "Are Musicians 'Ordinary Workers'? Labor Organization and the Question of 'Artistic Value' in the First Years of the Portuguese Musicians' Class Association: 1909-1913”, Popular Music and Society, XL/5, pp. 518-38, DOI: https://doi.org/10.1080/03007766.2017.1348671.

Vera, Fernanda

2015 “Músicos sin pasado? Construcción conceptual en la historiografía musical chilena”. [tesis de Magíster en artes mención Musicología], Universidad de Chile.

Williamson, John Y Martin Cloonan

2016 Players'work time: a history of the British Musicians'Union, 1893-2013. Manchester: Manchester University Press.

Witker, Alejandro

1977 Los trabajos y los dias de Recabarren. La Habana: Casa de las Américas.

\section{Entrevistas}

TORRES, RODRIGO

2017 Entrevistado por Eileen Karmy, 4 de mayo, Santiago de Chile.

\section{Legislación}

DiARIO OFICIAI

1925 Ley 345, Santiago de Chile, 17 de marzo. 